\title{
Article \\ Computational Fluid Dynamic Analysis of Flow Rate Performance of a Small Piezoelectric-Hydraulic Pump
}

\author{
Phuc Nguyen Anh, Jae-Sung Bae and Jai-Hyuk Hwang * \\ School of Aerospace and Mechanical Engineering, Korea Aerospace University, Goyang-si 10540, Korea; \\ phucabc@kau.kr (P.N.A.); jsbae@kau.ac.kr (J.-S.B.) \\ * Correspondence: jhhwang@kau.ac.kr; Tel.: +82-02-300-0109
}

Citation: Anh, P.N.; Bae, J.-S.; Hwang, J.-H. Computational Fluid Dynamic Analysis of Flow Rate Performance of a Small Piezoelectric-Hydraulic Pump. Appl. Sci. 2021, 11, 4888. https://doi.org/ 10.3390/app11114888

Academic Editor: Shujun Zhang

Received: 10 April 2021

Accepted: 25 May 2021

Published: 26 May 2021

Publisher's Note: MDPI stays neutral with regard to jurisdictional claims in published maps and institutional affiliations.

Copyright: (c) 2021 by the authors. Licensee MDPI, Basel, Switzerland. This article is an open access article distributed under the terms and conditions of the Creative Commons Attribution (CC BY) license (https:/ / creativecommons.org/licenses/by/ $4.0 /)$.

\begin{abstract}
This paper investigates the transient flow rate performance of small piezoelectric-hydraulic pumps. In a previous study, a small piezoelectric hydraulic pump was designed and developed to be applicable to the braking systems of small- and medium-sized UAVs (unmanned aerial vehicles). To this end, a thin plate spring check valve was designed in order to effectively discharge the flow in a single direction. The flow rate of the piezoelectric-hydraulic pump is an important criterion for evaluating pump efficiency. Therefore, a study on the parameters affecting such a flow rate is necessary to enhance the efficiency of piezoelectric hydraulic pumps used in brake systems. This study on small piezoelectric-hydraulic pumps is performed to accurately predict the flow rate using a CFD (Computational Fluid Dynamics) tool. In other words, an unsteady CFD method is applied to model the transient flow rate characteristics and the internal flow field of the fluid. The visualization of the internal flow field is evaluated for a better understanding of the flow fields inside the pump. Moreover, this work also illustrates the detailed motion of both the inlet and outlet check valves during the pump operation that fully reflects the phase shift between the check valves and the piston motion, all of which affect the flow rate performance of the pump. An experiment of flow rate characteristics was conducted on a designed piezoelectric-hydraulic pump, which verifies the validity of the CFD results.
\end{abstract}

Keywords: piezoelectric actuator; check valve; phase shift; CFD; flow field

\section{Introduction}

With advantages such as low costs, supply safety, reliability, and structural simplicity, hydraulic systems with power oil pressure have a lot of potential upsides when being applied to aircraft as a mechanical power transmission. Hydraulic systems are often used in the braking system which plays a pivotal role in stopping the aircraft's movement. With a complex structure that includes many parts such as a pressure accumulator, solenoid valves, a hydraulic reservoir, check valves, and electronic control devices, the hydraulic pump used in aircraft brake systems commonly possesses great size and weight. This is one of the disadvantages of the conventional hydraulic system used in unmanned aircraft, which have stricter weight and space constraints. Therefore, a miniaturization of the brake system which is applicable to small- and medium-sized UAVs requires a small-sized hydraulic pump that forms the flow rate and load pressure. Currently, many researchers have carried out research to design smaller-sized smart material pumps [1,2], and therefore, several types of smart material actuators such as IPMCs (ionic polymer-metal composites), SMA (shape memory alloy), and PZT (lead zirconate titanate) have been investigated for their wide application [3-5]. As the piezostack actuator could create a large load pressure corresponding to a small displacement, it is the most relevant choice for the development of brake systems used in small- to medium-sized unmanned aircraft. Recently, more attention has been focused on membrane actuators, coined "piezo membrane pumps", for medium delivery in order to study piezoelectric micropumps, e.g., the valve-less pumps [6-8], check 
valve pumps [9-11], and peristaltic pumps [12-14] with its benefits of compact structure and volume. Besides, some scholars have approached the problem differently by utilizing piezostack actuators $[15,16]$ called piezoelectric-hydraulic pumps, or piezostack pumps. For high-power applications, the piezoelectric-hydraulic pumps are more relevant and possess the ability to obtain higher backpressure, especially for the piezoelectric-hydraulic linear actuators compared to the piezo membrane pumps. The design and fabrication of a small piezo-electric hydraulic pump with a PZT actuator applied to UAV brake systems have been proposed to demonstrate superior performances, i.e., low power consumption, high reliability, and precise pressure control in comparison with its conventional counterparts.

To satisfy the brake system requirements of small- and medium-sized UAVs, e.g., the delivered flow rate, fast response speed, high pressure, and stability during its operation, a small piezoelectric-hydraulic pump was proposed [17]. Piezoelectric ceramic actuators have the potential to generate greater force and work at high speeds with small deflection compared to other smart materials. It is, therefore, necessary to design a relevant check valve that can release the small flow rate of the piezoelectric-hydraulic pump to ensure a single-direction flow. In this study, a thin-plate spring check valve was proposed to transmit the small flow rate in the output direction, which allows the check valve to open more easily whenever there is a pressure variation on its two sides. Also, the valve has the capability of operating well under the high-speed vibration of the piezo-ceramic actuator. Furthermore, being a passive type, the check valve can lessen the complexity of the system to satisfy the lightweight and miniaturization standards. Despite the delivered flow rate being relatively small [18-20], the piezoelectric-hydraulic pump can increase the driving frequency to reach the required flow rate. A formula to calculate flow rate performance has been developed [21]. The results obtained from the calculated formula are quite accurate and consistent with the experimental results. However, evaluating the flow velocity as well as the pressure inside the pump during operation is impossible, so a complete simulation of the pump is essential.

Up until recently, most of the research has focused on pump flow results, while other factors of flow such as velocity and flow direction have not been adequately studied. Moreover, the above studies consider the force acting on the check valve as a sine function, but in fact, due to the influence of the movement of the check valve, this force can change over time. This is known as the Fluid-Structure Interaction (FSI) problem. Therefore, a study of the parameters affecting such a flow rate is necessary to enhance the effectiveness of the piezoelectric hydraulic pump used in the brake system. The flow rate of the piezoelectrichydraulic pump is of interest to satisfy the requirements of the brake system. In this paper, a transient CFD simulation on both the inlet and outlet check valves during the operation was performed. The transient simulation describes the motion of both check valves and the flow field inside the pump, which is difficult to determine in the experiment. In other words, an unsteady CFD method is used to model the transient flow rate characteristics and the internal flow field of the fluid. The visualization of the internal flow field evolution is considered for a better understanding of the flow fields inside the pump. Furthermore, this paper illustrates a detailed motion of both check valves opening during the pump operation. The experiment on flow rate characteristics was conducted on a developed piezoelectric-hydraulic pump, which verifies the validity of the CFD results.

\section{Working Principles of the Piezoelectric-Hydraulic Pump Brake System}

In this paper, the designed piezoelectric-hydraulic pump brake system includes a piezostack actuator, two check valves, a piston, and a chamber space, all of which are presented in Figure 1. When the PZT actuator of the piezoelectric-hydraulic pump is applied by changing the voltage, the mechanical energy can be transferred into liquid flow. 


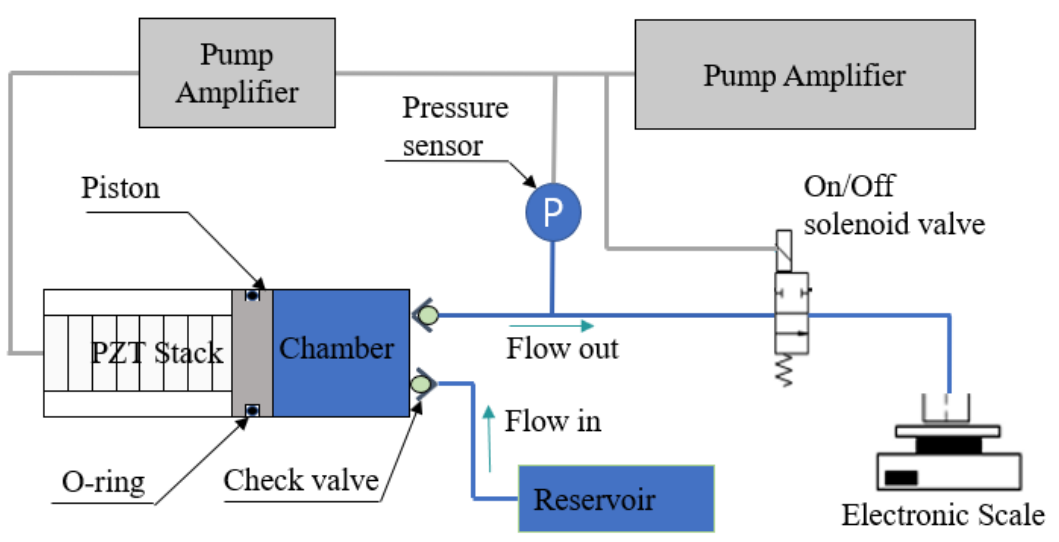

Figure 1. Schematic of the piezoelectric-hydraulic pump brake system.

Table 1 represents the specifications of the PZT actuator used in the piezoelectric hydraulic pump. A piston is in the chamber transmission part which connects the liquid and the PZT actuator. When the PZT actuator is operating in the extending-shrinking vibration mode, the compressed liquid in the chamber allows the check valve to alternately open and close. Consequently, the liquid of the system is continuously transferred from the inlet into the outlet.

Table 1. Operating parameters of the PZT actuator.

\begin{tabular}{ccc}
\hline Piezo Stack-Model P-225.40 & Value & Unit \\
\hline Operating voltage & $0-1000$ & $\mathrm{~V}$ \\
Closed-loop travel & 60 & $\mu \mathrm{m}$ \\
Unload resonant frequency & 7 & $\mathrm{kHz}$ \\
Push/pull force capacity & $12,500 / 2000$ & $\mathrm{~N}$ \\
Mass (with cable) & 610 & $\mathrm{~g}$ \\
\hline
\end{tabular}

The type of check valve set up for the piezoelectric hydraulic pump is the thin-plate spring check valve. It can effectively operate with the high-speed oscillation of the PZT actuator. The check valve holds an important role in the hydraulic pump and was created to adequately prevent fluid back-flow. The thin-plate spring check valve (Figure 2) consists of bending cantilevers, the clamping boundary, and the valve plate which prevents reverse flow. The displacement of the check valve can be adjusted by changing the shape, size, or material of the bending cantilevers. In this research, stainless steel was used for making the check valve with a chosen thickness of $0.2 \mathrm{~mm}$. An O-ring was applied to prevent reverse flow and to create a gap between the pump seat and the check valve. The structure of that gap causes the liquid environment around the valve to change. Therefore, it is necessary to take into account the effect of the gap on the valve operation when the simulation was created.

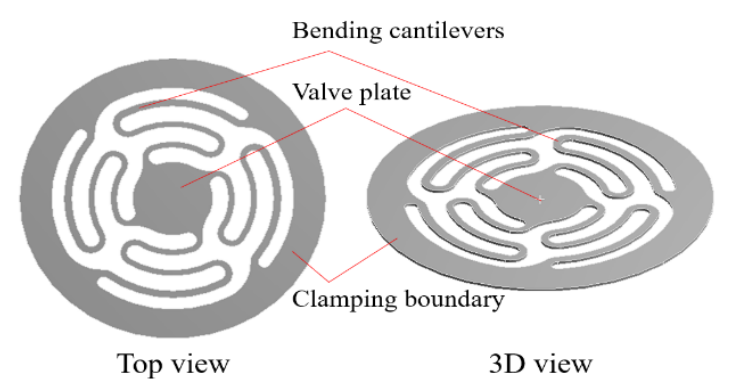

Figure 2. Thin-plate spring-type check valve. 


\subsection{The Force Applying on the Check Valve Operating in Fluid}

When the PZT actuator oscillates, the force acts on the check valve by the flow rate generated by the PZT actuator. However, the check valve will oscillate which in turn leads to the boundary conditions changing over time. Accordingly, the force acting on the check valve will be determined by an FSI interaction simulation by using the Ansys program. As mentioned above, the oscillation of the thin-plate spring check valve is in the liquid domain. The added liquid mass and added damping, therefore, are considered. The dynamic equation of the check valve operating in the fluid domain can be written as:

$$
M \ddot{y}+C \dot{y}+K y=F(t)
$$

where $M, C, K$ and $F$ refer to the total effective mass of the valve in a fluid environment ( $M=M_{v}+M_{a}$, where $M_{v}$ represents the effective mass of the check valve, and $M_{a}$ is the added mass of liquid), the added damping, the stiffness of the check valve, and the driving force generated by the PZT actuator, respectively.

In the piezoelectric-hydraulic pump, the liquid flow is driven by the oscillating actuator which opens and closes the check valve repeatedly. At the same time, the fluid reversely holds the main role in resistance to the check valve oscillation. The actuator oscillation, the check valve movement and the liquid are thus coupled. The fluid reaction force is referred to as an added mass and the added damping contributes to the dynamic response of the check valve without changing its stiffness. Assuming the fluid to be incompressible, the driving force generated by the actuator will be forced to the check valve the right way. Consequently, the external force, $F(t)$, can be separated into two contributions:

$$
F(t)=F_{\text {reaction }}(t)+F_{\text {drive }}(t)
$$

the first term, $F_{\text {reaction }}(t)$ in Equation (2), is a reaction loading component caused by the motion of the fluid around the check valve, while the second term, $F_{\text {drive }}(t)$, refers to a driving force that excites the check valve. This force is usually a known function of time.

Related to fluid-structure interaction, the hydrodynamic loading, which is mainly generated due to the pressure drag [17], has been separated by two uncoupled terms:

$$
F_{\text {reaction }}(t)=-M_{a} \ddot{y}-C_{a} \dot{y}
$$

where $M_{a}$ represents the added mass per unit length and $C_{a}$ refers to the added damping coefficient. The negative sign in Equation (3) demonstrates that the hydrodynamic forces always reverse the check valve motion. Substituting Equation (3) into Equation (2), we get an uncoupled equation of the check valve's motion:

$$
\left(M_{v}+M_{a}\right) \ddot{y}+\left(C_{v}+C_{a}\right) \dot{y}+K y=F_{\text {drive }}(t)
$$

where the mass and damping coefficients contain the contributions from the check valve and the ambient liquid.

The purpose of the numerical simulation, which considers the check valve and surrounding liquid, is to identify the response of the check valve in a fluid domain. It is possible to observe the simultaneous fluctuation between the check valve and piston during pump operation. From that simulation, it is possible to obtain some important characteristics such as the flow value, the flow velocity, the valve's oscillation, as well as the pressure in the chamber. The ANSYS program is also used to simulate the oscillation of the check valve and to provide internal flow field evolutions as a visualization for a better understanding of the pressure and flow fields inside the pump.

\subsection{Effect of Phase Shift between the Piston and Check Valves on the Flow Rate Performance}

Each cycle oscillation of the PZT actuator is separated into 2 processes: one is pushing fluid out of the chamber (from $t_{0}$ to $t_{1}$ in Figure 3 ), and another is pulling fluid into the chamber (from $t_{1}$ to $t_{2}$ in Figure 3). With the phase shift at 0 (Figure 3a), the PZT 
actuator pushes the fluid out of the chamber, whereas the inlet check valve closes and the outlet check valve opens. This results in all fluid exiting through the outlet check valve. Otherwise, when the PZT actuator pulls the fluid out of the chamber, the inlet check valve opens and the outlet check valve closes. This means that the whole fluid flows into the chamber through the inlet check valve. Consequently, the fluid continually flows from inlet to outlet and the volume of flow obtained at the outlet is at its greatest. In the case of the phase shift increasing to $\pi / 2$ (Figure $3 b$ ), the entering and exiting liquid flows equally through each check valve in each process. Therefore, the total flow rate through the outlet check valve is zero. Contrary to the first case, the fluid will flow into the outlet and discharge through the inlet when the phase is close to $\pi$ (Figure 3c). This leads to the reverse flow coming forth.

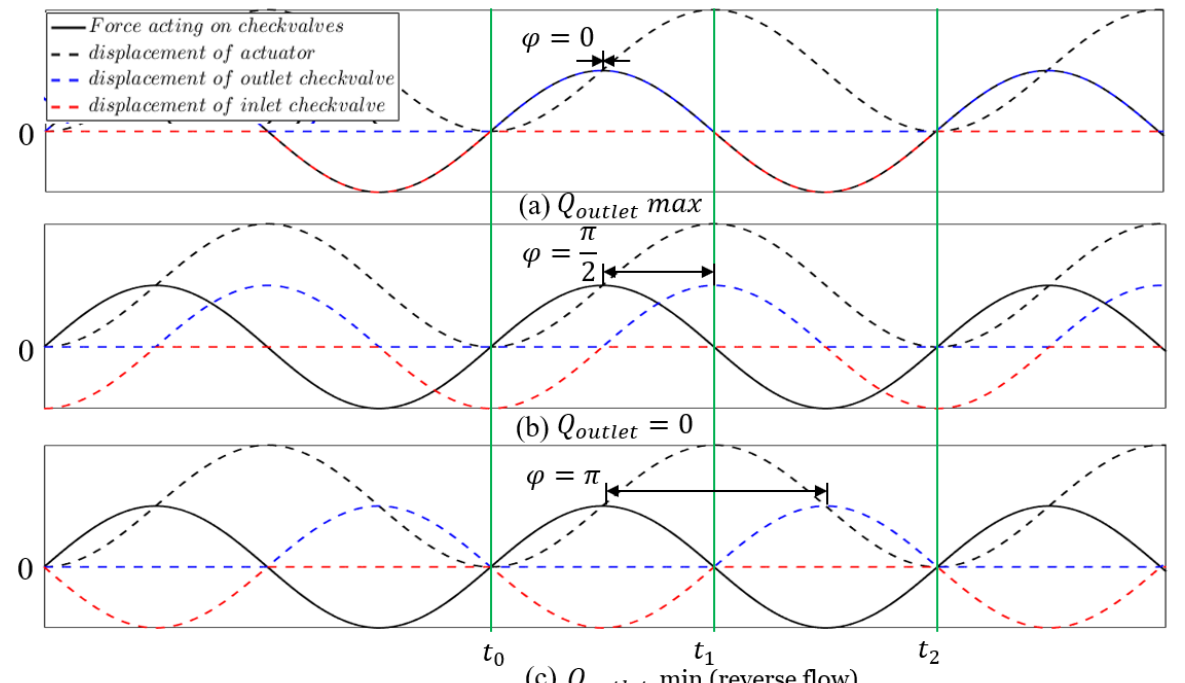

(c) $Q_{\text {outlet }} \min$ (reverse flow)

Figure 3. The assumed relationship between the displacement of the PZT actuator and the check valves (scaled). (a) phase shift $\varphi=0$, (b) phase shift $\varphi=\pi / 2$, (c) phase shift $\varphi=\pi$.

The example above helps to estimate the effect of the phase shift between the valve and the piston motion on the amount of fluid exiting from the outlet. When the driving frequency of the piston oscillation increases, the phase shift value also increases. This result has been verified in previous studies [21].

\section{CFD Simulation Methodology}

For modeling in this research, given that the motion of the check valve is an interaction of structural body and fluid, we need to model this problem as an FSI simulation, shown in Figure 4. In order to do so, we chose the widely used CFD commercial software ANSYS Fluent, which is a good way of simulating fluid-body interaction, to implement mesh and the numerical simulation in this paper. To attain a totally developed flow inside the studied pump, simultaneous movement of both the inlet check valve and outlet check valve along with the piston motion is considered in this study. Because the flow through the check valve can flow in two directions: entering and exiting the chamber, the outlet and inlet boundary type are assigned as pressure outlet and pressure inlet, which are set up to be at atmospheric pressure, since the concern of this research is the pressure difference. 


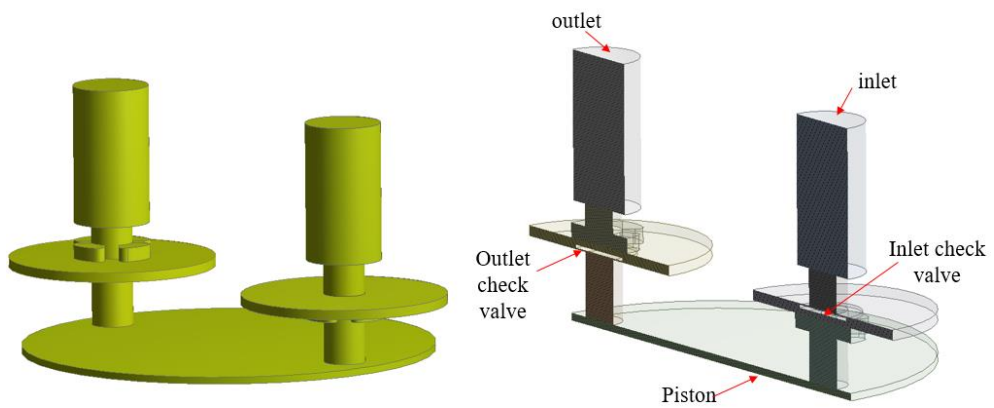

Figure 4. The fluid domain inside the piezoelectric-hydraulic pump.

The grid simulation is defined as a trimmed mesher (predominantly hexahedral mesh), and the refinement of the boundary layer is defined as a prism layer mesher. Local grid refinement is conducted in the area near the check valve where the internal flow field is more complicated. In other words, refinement mesh is applied in areas near the movement zone of the check valve, and the piston where velocity gradients are predicted. A finer mesh tool is applied, with the size of the mesh at that zone being $0.025 \mathrm{~mm}$. The grid quality is checked thoroughly. The refinement mesh of the important region is shown in Figure 5.

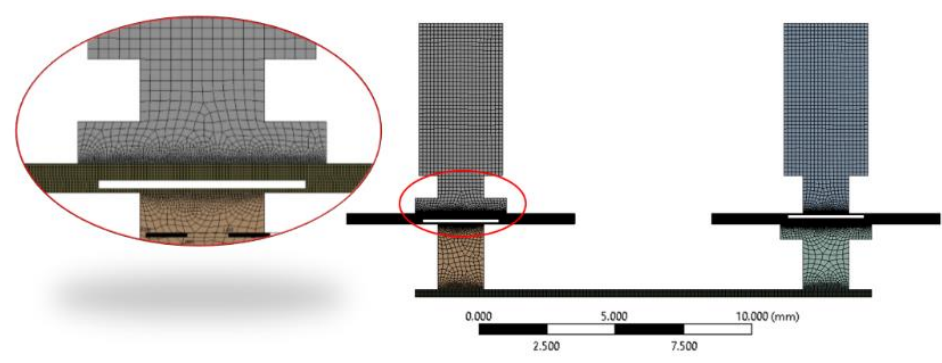

Figure 5. Mesh of the fluid inside the piezoelectric hydraulic pump.

For the overall mesh size, a convergence test is conducted to determine the size of elements in the modeling for computational accuracy and efficiency. In order to obtain the optimal solution, different mesh sizes comprising of $2 \mathrm{~mm}, 1.5 \mathrm{~mm}, 1 \mathrm{~mm}, 0.5 \mathrm{~mm}$, and $0.25 \mathrm{~mm}$ are used in the convergence test. The calculated flow rate at the driving frequency of $150 \mathrm{~Hz}$ of the studied pump corresponding to the different mesh sizes is represented in Figure 6. It can be seen from the figure that the flow rate becomes converged when the overall mesh size is $0.5 \mathrm{~mm}$. Further reducing the mesh size does not considerably affect the predicted flow rate, but does significantly increase the computational cost. The overall mesh size of $0.5 \mathrm{~mm}$ is, therefore, utilized in the subsequent investigations.

For the transient FSI-simulations, the boundary of the check valve will change. This requires a method that treats the mesh movement. In this study, the moving direction is known, which in turn allows the dynamic layering method to be used. The dynamic mesh capability is used to model problems with check valves and piston motion. There are commonly three dynamic mesh schemes, namely layering, smoothing, and re-meshing within ANSYS Fluent. A combination of these three schemes is applied to tackle the most challenging dynamic mesh problems. In this case, however, the layering scheme is often sufficient. Check valves are often used to allow unidirectional flow. They are usually applied to act as a pressure-relieving device by allowing fluid to be pushed out of the domain when the pressure is higher than a certain level. In such a case, check valves are connected to the bending cantilever (acting as a spring) that acts to push the valve close to the pump seat and to shut off the flow. However, when the force on the valve is larger than the restoring force, the valve will move away from the pump seat and allow fluid to discharge, thus reducing the pressure upstream inside the chamber. The deformation of the valve plate is typically neglected. 


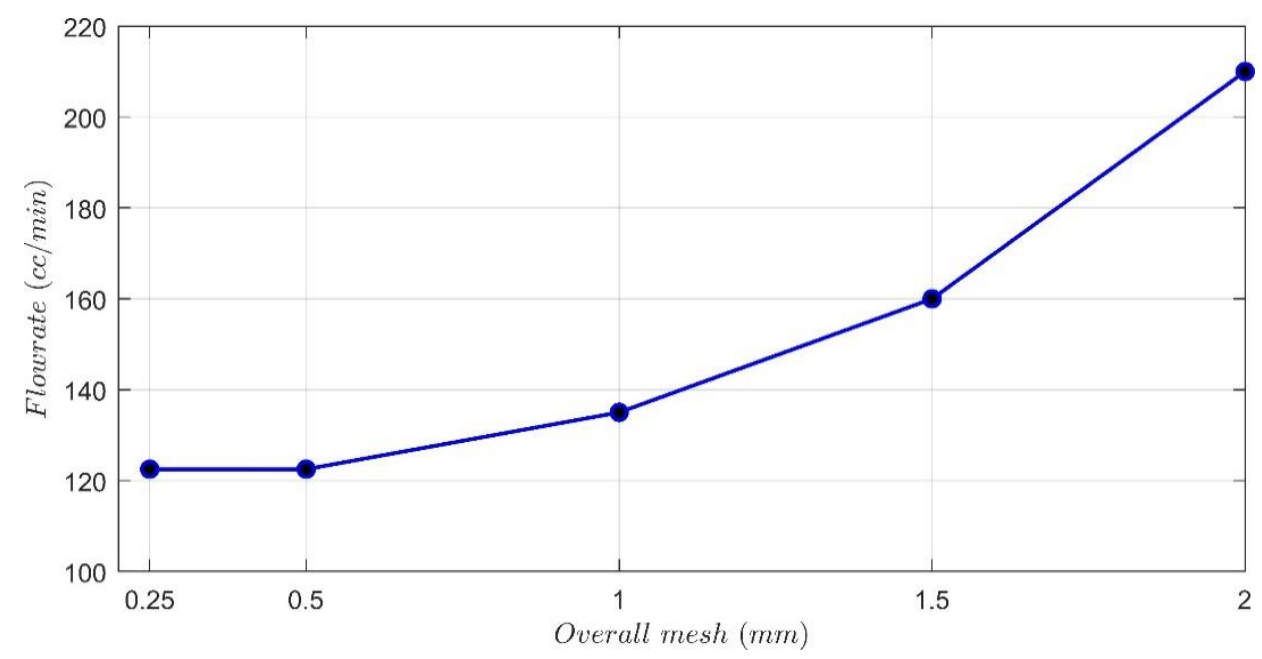

Figure 6. Effect of mesh sensitivity on the flow rate.

In this study, Mil-PRF-83282, which is commonly used in aircraft hydraulic brake systems, was employed as the hydraulic fluid with a density of $\rho=851 \mathrm{~kg} / \mathrm{m}^{3}$ and a dynamic viscosity of $\mu=8.88 \times 10^{-4}$ Pa.s at $20^{\circ} \mathrm{C}$. The realizable $\mathrm{k}-\varepsilon$ model, a common turbulence model, was adopted for the turbulence simulation. The pressure-based coupled algorithm is used to deal with a coupled system of equations including the pressure-based continuity equation and the momentum equations. Similar to the mesh convergence test, a time step is set to be $0.0001 \mathrm{~s}$, since the flow rate barely changes as the time step reduces. The input motion of the piston is a User-Defined Function (UDF) used to describe the simulation. When a sinusoidal input voltage is applied to the PZT actuator, the piston attached to the actuator also has the sinusoidal motion, with the maximum amplitude dependent on the amplitude of the supplied voltage. In this study, the maximum applied voltage is $900 \mathrm{~V}$, and the corresponding amplitude of piston motion is $54 \mu \mathrm{m}$.

\section{Numerical Simulation Results}

\subsection{Flow Field Analysis of the Piezoelectric-Hydraulic Pump}

Figure 7 shows the flow field of the part around the check valve and inside the chamber at some typical moments. The vortex near the corner of the pump seat and flow velocity change are both generated by the abrupt expansion of the flow channel at the pump seat shoulder and the valve plate. This is a result of the high-velocity flow passing from the inlet through the outlet via a narrow flow passage. When the actuator operates at a large frequency of oscillation, the flow has a higher velocity, resulting in a larger force applying to the check valve. Thus, in the case of the high driving frequency, the pressure and velocity of the flow in the chamber are larger than when it operates at small frequencies.

Besides flow velocity and chamber pressure, the flow rate and piston movement can also be calculated with the above simulation. The obtained flow rate at a driving frequency of $120 \mathrm{~Hz}$ was shown in Figure 8. The latter will be shown in more detail in the next section. 

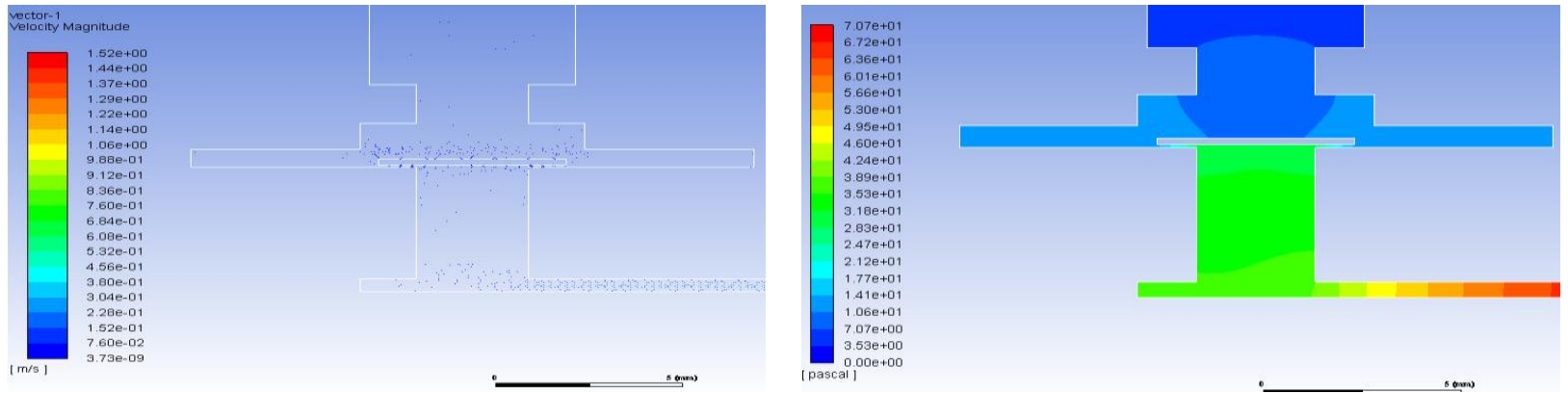

(a)

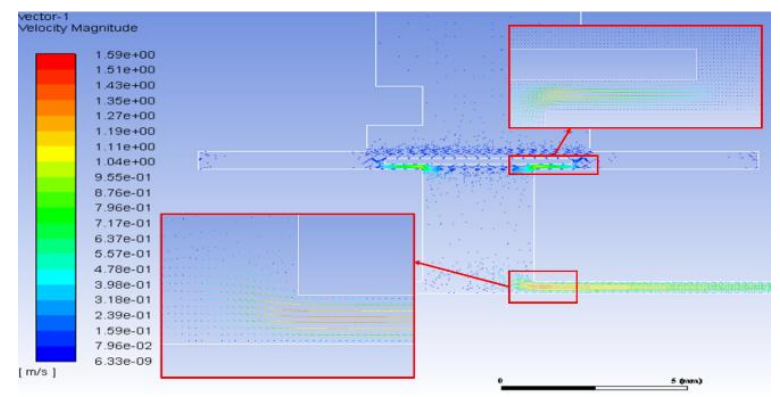

(c)

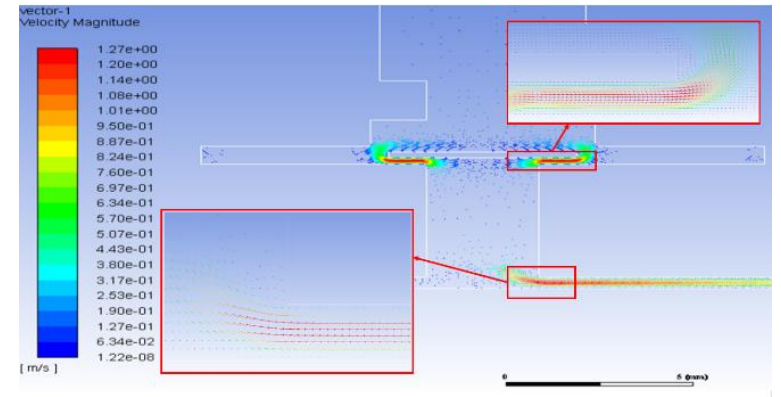

(e)

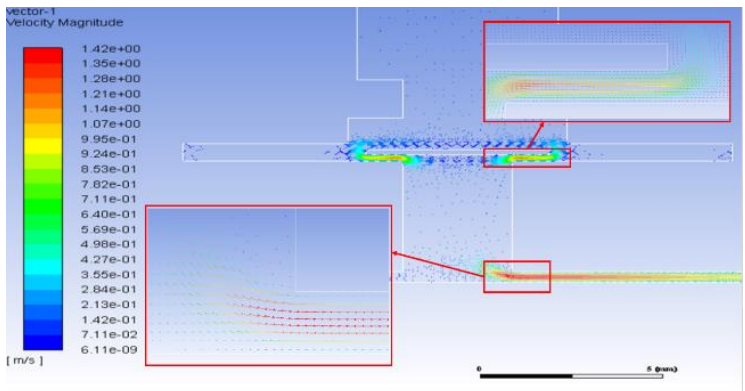

(g) (b)

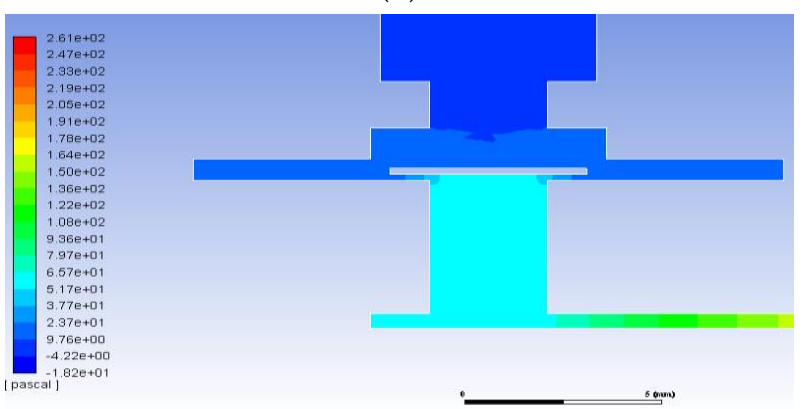

(d)

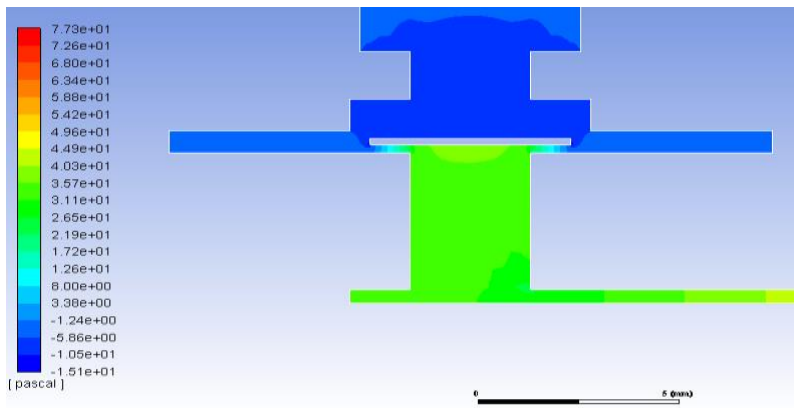

(f)
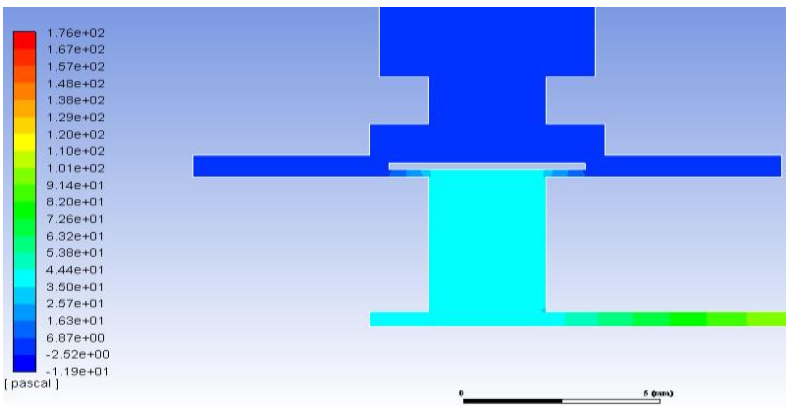

(h)

Figure 7. Flow field results at the outlet check valve at a driving frequency of $120 \mathrm{~Hz}$ at some typical moments. (a) Velocity vector at $t=2 \times 10^{-4} \mathrm{~s}$; (b) Pressure contour at $t=2 \times 10^{-4} \mathrm{~s}$; (c) Velocity vector at $t=4.16 \times 10^{-4} \mathrm{~s}$; (d) Pressure contour at $t=4.16 \times 10^{-4} \mathrm{~s} ;(\mathbf{e})$ Velocity vector at $t=1.04 \times 10^{-3} \mathrm{~s} ;(\mathbf{f})$ Pressure contour at $t=1.04 \times 10^{-3} \mathrm{~s}$; (g) Velocity vector at $t=2.08 \times 10^{-3} \mathrm{~s} ;\left(\right.$ h) Pressure contour at $t=2.08 \times 10^{-3} \mathrm{~s}$. 


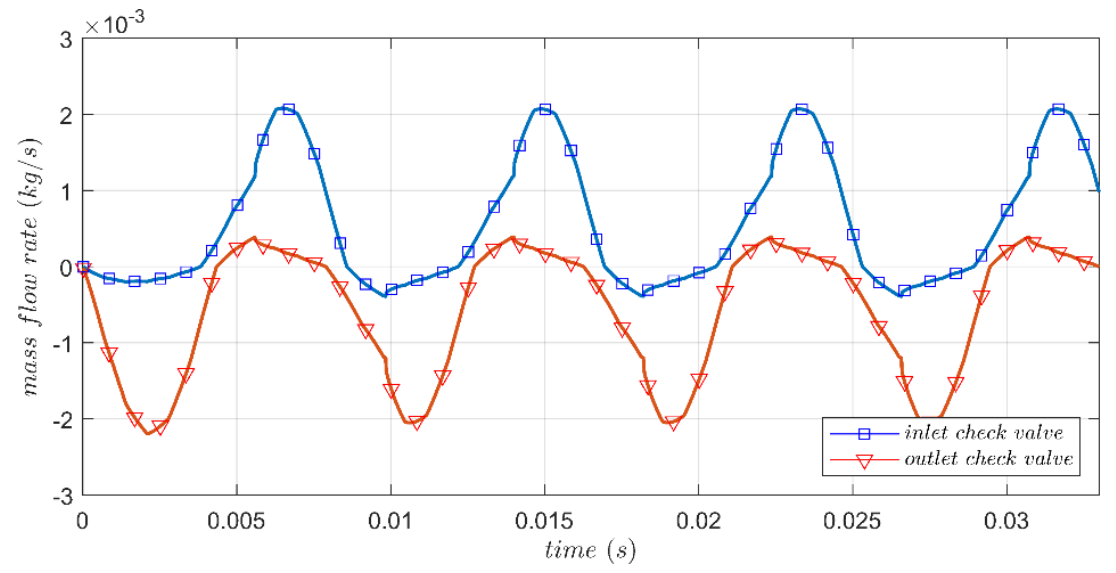

Figure 8. The transient flow rate result at $120 \mathrm{~Hz}$.

\subsection{Check Valve Motion Characteristics}

The difference in the movement of the check valves can be seen when the oscillation frequency of the actuator changes. In the previous example on flow rate performance of the piezoelectric-hydraulic pump in Section 2.2, the effect of phase shift on the opening and closing process of the check valves is carefully described. However, as mentioned earlier, the interaction between the check valves and the fluid is an FSI problem, which is nonlinear. In turn, assuming that the check valves' movements in a fluid medium follow a sinusoid form [21], would not be completely correct as in practice. The FSI simulation in Ansys will generate the result of the displacement between the two valves in the most realistic way. The obtained movement of the inlet check valve and outlet check valve will provide a better understanding of the effect of the phase shift on the results of the obtained flow rate. The movements of both check valves corresponding to the driving frequency domain of the actuator are shown in Figure 9.

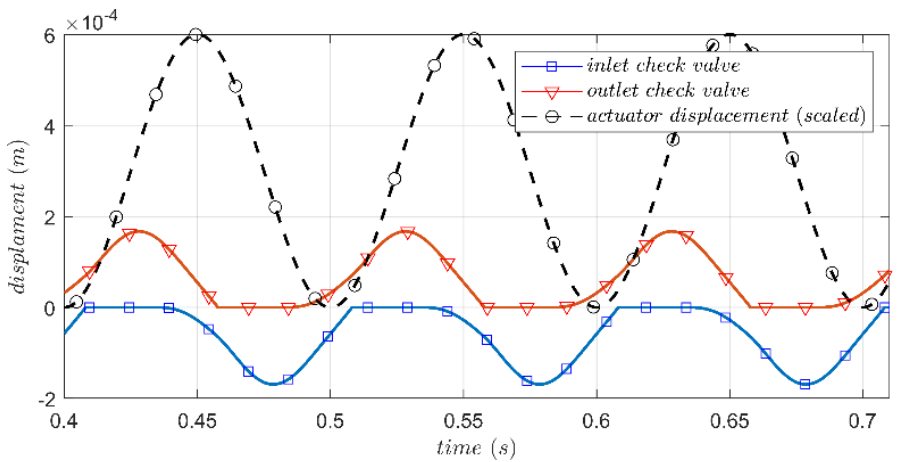

(a)

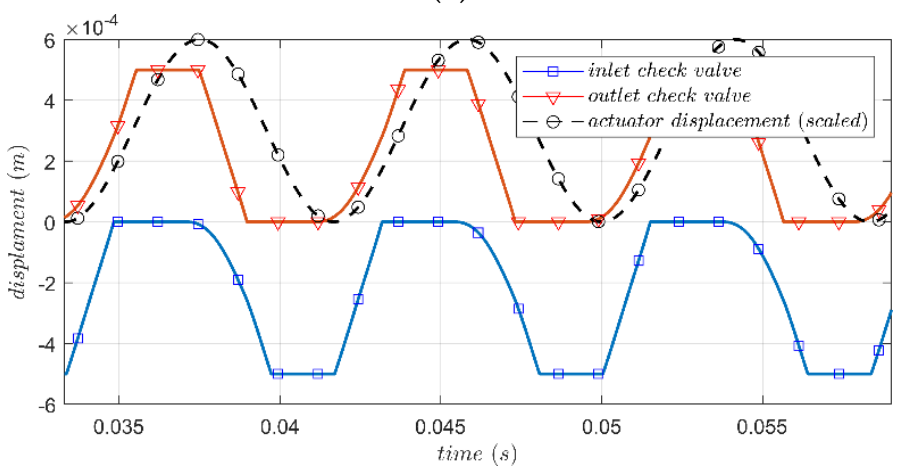

(b)

Figure 9. Cont. 


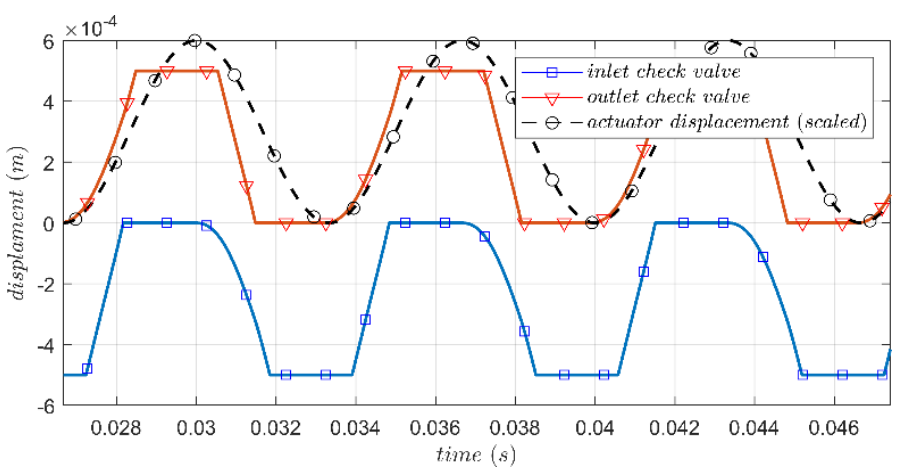

(c)

Figure 9. The displacement of check valves at several driving frequencies. (a) Driving frequency: $10 \mathrm{~Hz}$. (b) Driving frequency: $120 \mathrm{~Hz}$. (c) Driving frequency: $150 \mathrm{~Hz}$.

The difference between the flow obtained at the low and the high operating frequency of the piston comes from the phase shift value between the valve movement and the piston, clearly exemplified in Section 2.2. At low frequencies, the inlet and outlet alternately open and close when the piston moves up and down, respectively. However, at high frequencies, the regular opening and closing in this way are no longer available. Although the piston goes up, the inlet check valve can be opened, and the outlet check valve can be closed. Due to this phenomenon, the amount of fluid flowing through the inlet and the outlet changes.

The result of the total flow pumped out is shown in Figure 10. The linear relationship between the total flow and the frequency of the piston can be seen at low frequencies. However, at high frequencies, this relationship changes. The greater the frequency, the lower the flow value. This is explained by the fact that, when the actuator operates at a high frequency, the opening and closing of the inlet and the outlet check valves changes. When the piston goes up, the liquid is ejected and the inlet and outlet open. This reduces the amount of liquid exiting the outlet, which in turn reduces the obtained flow rate.

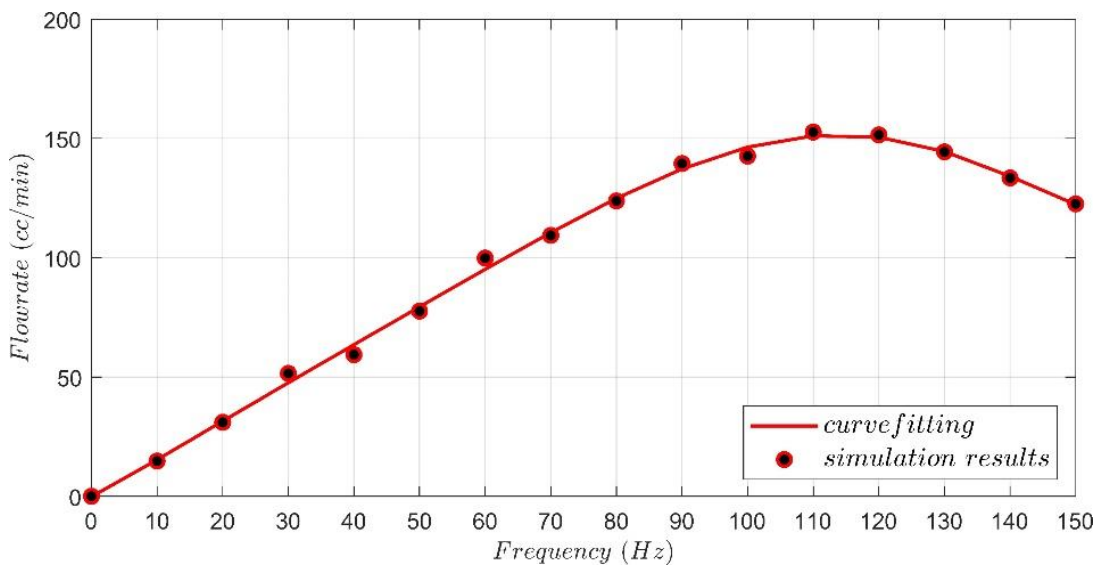

Figure 10. The flow rate simulation result at several driving frequencies.

\section{Experiment Verification for the Flow Rate of the Piezoelectric-Hydraulic Pump}

In order to validate the results from the computational simulation, an actual piezoelectrichydraulic pump for the UAV brake system is fabricated and represented in Figure 11. The piezoelectric-hydraulic pump components were designed and made for the easiest removal and replacement. Most of these components were manufactured by precision machining. However, the check valve was made using wire-cut electric discharge machining, since it has a complex shape and thin plate. 


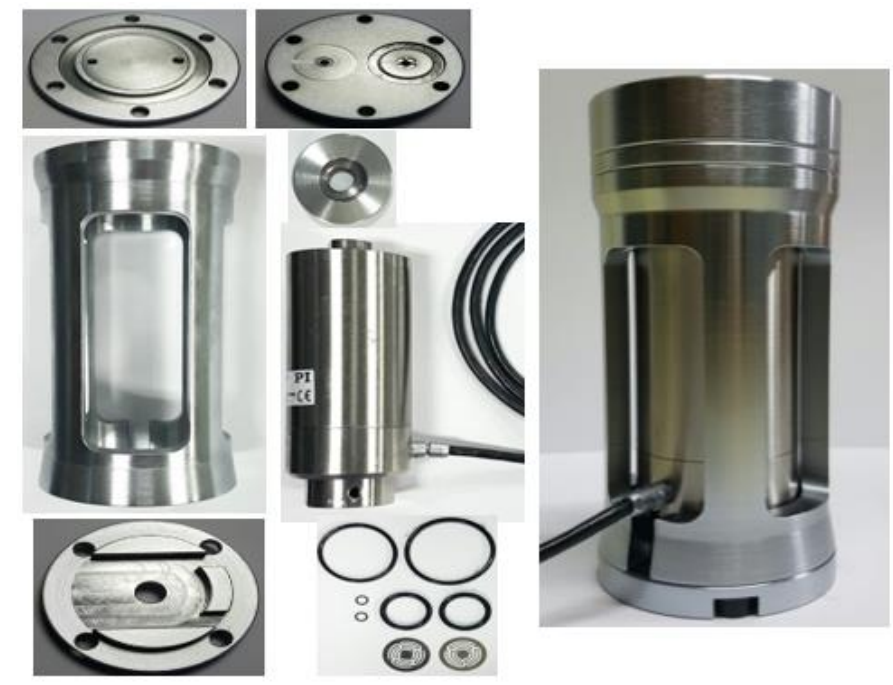

Figure 11. Components of the designed piezoelectric-hydraulic pump used in the experiment.

In addition, to fabricate a piezoelectric-hydraulic pump, some important equipment is required to conduct the experiment in order to reinforce the results from the simulation, such as a DAQ program, a pressure sensor, a solenoid valve, a high-voltage amplifier, and an electronic scale, as shown in Figure 1. The high-voltage amplifier is used to amplify the created signal and then applies that signal to the piezoelectric actuator at a desired operating frequency. The DAQ program "dSPACE" allows the collection and creation of the digital and analog signal data.

The solenoid valve was linked to the exhaust port of the piezoelectric-hydraulic pump and made a major contribution to the exhaust performance. When the solenoid valve was closed, a closed-loop hydraulic circuit, such as that in a brake system, was formed. In addition, the flow rate in the no-load state was evaluated when the solenoid valve was opened. A pressure sensor was used to measure the pressure of the load side. As for the fluid, Mil-PRF-83282, commonly used in actual aircraft hydraulic brake systems, was used in this research. The flow rate experiment was conducted at several driving frequency values from 0 to $150 \mathrm{~Hz}$. The flow rate performance was collected and is shown in Figure 12.

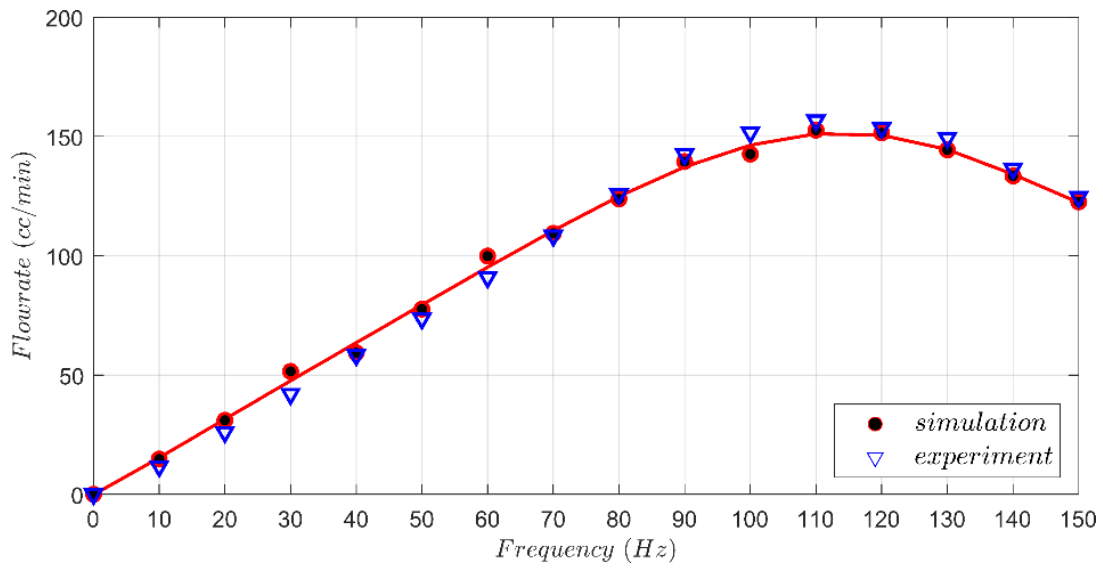

Figure 12. The flow rate experiment results at several driving frequencies.

A good match between the estimation method and the CFD simulation, which is shown in Figure 12, is guaranteed. Therefore, the estimation by using a simulation is effective for a coarse approximation of the check valve motion and flow rate performance of the piezoelectric hydraulic pump. 


\section{Conclusions}

In this paper, the flow rate performance of the small piezoelectric-hydraulic pump brake system was investigated. Due to the inclusion of simultaneous moving parts in liquid, the phase shift in motion is considered in this study to analyze and observe the flow inside the pump. The FSI simulation has been used to analyze the flow rate performance. The simulation results showed that when the actuator has a large driving frequency of oscillation, the flow has a higher velocity, leading to a larger force applying to the check valves. Therefore, in the case of high oscillation frequency, the pressure and velocity of the flow in the chamber are greater than when it operates at low frequencies where the relationship between the total flow rate and the driving frequency of the piston is linear. However, this relationship changes at high frequencies; the greater the frequency, the lower the obtained flow value. When the actuator operates at a high frequency, the phase shift between check valves and actuator makes the inlet check valve open, while the actuator goes up. In turn, some of the fluid will exit through the inlet instead of the outlet. This is one reason why the obtained flow rate reduces at high driving frequencies. The simulation evaluating the flow rate presents a good match with those generated from the experiment. When the driving force operates at a frequency of $110 \mathrm{~Hz}$, the maximum flow rate value is about $158 \mathrm{cc} / \mathrm{min}$. The obtained results also show that changing the driving frequency of the check valves will lead to the change in obtained flow rate value. In turn, the flow rate performance of the piezoelectric- hydraulic pump will be improved by optimizing the driving frequency, corresponding to the check valves being used. For small devices, studying vibrations in liquids is very difficult. Therefore, the simulation mentioned in this study should be very useful in research and in the manufacturing of such small devices. The simulation can be applied in cases with simultaneous moving parts in liquids to have a correct visualization and analysis of the flow.

Author Contributions: This study was initiated and designed by J.-H.H. The simulations were completed by P.N.A. The data analysis and write-up of the paper were completed by P.N.A. and J.-S.B. The suggestions for the simulation design and paper-writing were completed by J.-S.B. and J.-H.H. All authors have read and agreed to the published version of the manuscript.

Funding: This work was funded by the Ministry of Trade, Industry \& Energy (MOTIE, Korea), grant number 10073291.

Institutional Review Board Statement: Not applicable.

Informed Consent Statement: Not applicable.

Acknowledgments: This work was supported by the Technology Innovation Program (intelligent landing gear with variable damping force for 1500lb class) (10073291).

Conflicts of Interest: The authors declare no conflict of interest.

Ethical Issues: The proposal does not take the participation of any human labor or violate animal welfare. Therefore, there should be no ethical issues.

\section{References}

1. Larson, J.P.; Dapino, M.J. Reliable, high-frequency miniature valves for smart material electrohydraulic actuators. J. Intell. Mater. Syst. Struct. 2012, 23, 805-813. [CrossRef]

2. Cazorla, P.H.; Fuchs, O.; Cochet, M.; Maubert, S.; Le Rhun, G.; Robert, P.; Fouillet, Y.; Defay, E. Piezoelectric Micro-pump with PZT Thin Film for Low Consumption Microfluidic Devices. Procedia Eng. 2014, 87, 488-491. [CrossRef]

3. Laser, D.J.; Santiago, J.G. A review of micropumps. J. Micromech. Microeng. 2004, 14, R35-R64. [CrossRef]

4. Iverson, B.D.; Garimella, S.V. Recent advances in microscale pumping technologies: A review and evaluation. Microfluid. Nanofluidics 2008, 5, 145-174. [CrossRef]

5. Chaudhuri, A.; Wereley, N.M. Compact hybrid electrohydraulic actuators using smart materials: A review. J. Intell. Mater. Syst. Struct. 2011, 23, 597-634. [CrossRef]

6. Ullmann, A. The piezoelectric valve-less pump-performance enhancement analysis. Sens. Actuators A Phys. 1998, 69, 97-105. [CrossRef] 
7. Kim, H.-H.; Oh, J.-H.; Lim, J.-N.; Lim, K.-J.; Park, D.-H. Design of valveless type piezoelectric pump for micro-fluid devices. Procedia Chem. 2009, 1, 353-356. [CrossRef]

8. Zhang, J.-H.; Wang, Y.; Huang, J. Advances in Valveless Piezoelectric Pump with Cone-shaped Tubes. Chin. J. Mech. Eng. 2017, 30, 766-781. [CrossRef]

9. Kan, J.W.; Yang, Z.G.; Peng, T.J.; Cheng, G.M.; Wu, B. Design and test of a high-performance piezoelectric micropump for drug delivery. Sens. Actuators A Phys. 2005, 121, 156-161.

10. Fan, Z.; Liu, J.; Dong, J.; Li, J.; Jiang, B. Study on the large flow rate piezoelectric mini-pump with metal check valve. In Proceedings of the 2009 International Conference on Mechatronics and Automation, Changchun, China, 9-12 August 2009; pp. 4294-4298. [CrossRef]

11. Ren, Y.J.; Ma, Y.T.; Huang, D.; Chen, J.; Feng, J.H. Elastic string check valves can efficiently heighten the piezoelectric pump's working fre-quency. Sens. Actuators A Phys. 2016, 244, 126-132. [CrossRef]

12. Lin, Q.; Yang, B.; Xie, J.; Tai, Y.-C. Dynamic simulation of a peristaltic micropump considering coupled fluid flow and structural motion. J. Micromech. Microeng. 2006, 17, 220-228. [CrossRef]

13. Jang, L.-S.; Kan, W.-H. Peristaltic piezoelectric micropump system for biomedical applications. Biomed. Microdevices 2007, 9, 619-626. [CrossRef] [PubMed]

14. Liang, L.; Ma, X.; Zhang, T. Structure design and simulation of circular ring piezoelectric peristaltic pump. Trans. Chin. Soc. Agric. Eng. 2012, 28, 40-44.

15. Li, B.; Chen, Q.; Lee, D.G.; Woolman, J.; Carman, G.P. Development of large flow rate, robust, passive micro check valves for compact piezo-electrically actuated pumps. Sens. Actuators A Phys. 2005, 117, 325-330. [CrossRef]

16. Choi, S.; Yoo, J.; Cho, M.; Lee, Y. Position control of a cylinder system using a piezoactuator-driven pump. Mechatronics 2005, 15, 239-249. [CrossRef]

17. Hwang, J.-H.; Bae, J.-S.; Hwang, Y.-H.; Kwon, J.-Y. Pressurization Characteristics of a Piezoelectric-Hydraulic Pump for UAV Brake Systems. Int. J. Aeronaut. Space Sci. 2018, 19, 776-784. [CrossRef]

18. Hwang, J.H.; Bae, J.S.; Yang, J.; Kwon, J.Y. The design on the spring sheet type check valve of smart material hydraulic pump. In The Society for Aerospace System Engineering, Fall Conference Paper; The Society for Aerospace System Engineering: Daejeon, Korea, 2014; p. 85.

19. Hwang, L.J.; Yang, J.; Joo, Y.; Bae, J.S.; Kwon, J.Y. Design of the compound smart material pump for brake system of small and medium size UAV. J. Aerosp. Syst. Eng. 2015, 9, 1-7.

20. Lee, H.J.; Hwang, J.H.; Bae, J.S.; Kwon, J.Y. Conceptual design of compound smart material pump with sequential operation of fluid displacement-force. In The Society for Aerospace System Engineering, Spring Conference Paper; The Society for Aerospace System Engineering: Daejeon, Korea, 2014; p. 95.

21. Nguyen, A.P.; Hwang, J.H.; Hwang, J.H.; Bae, J.S. Effect of Check Valve Characteristics on Flow Rate of the Small PiezoelectricHydraulic Pump. J. Aerospace Syst. Eng. 2019, 12, 54-68. 